\title{
A Distinguishing Method of the Ultrasonic Signal Waveform Starting Point
}

\author{
Jiao Bing,Chen Xiaoying \\ Institute of Meteorology \\ PLA Univ. of Sci. \& Tech. \\ Nanjing, China, 211101
}

\author{
Ye Song,Wen Yating \\ Institute of Meteorology \\ PLA Univ. of Sci. \& Tech. \\ Nanjing, China, 211101
}

\begin{abstract}
According to the application of ultrasonic in wind speed, flow and liquid level measurement, a method about distinguishing ultrasonic signal waveform starting point was introduced. The high-powered DSP chip was chosen as the system's processor. The IIR band-pass digital filter was designed to eliminate disturbance. Marked change in fluctuation of ultrasonic signal sample was checked using the hypothesis testing to find the jumping off point of signal waveform.
\end{abstract}

Keywords-ultrasonic, digital filtering, hypothesis testing, DSP microprocessor

\section{INTRODUCTION}

Ultrasonic measurement method has many advantages such as the detecting element measurement that non-touched medium, wide measuring range, the low-temperature medium measurement, long service life, convenient maintenance, fixed-point measurement and continuous measurement.

Ultrasonic is widely used in wind speed measurement, flow measurement and liquid level measurement at present. Time difference method applied in wind speed and flow measurement[1], ultrasonic echo signal location method applied in liquid level measurement[2] could obtain the wind speed, flow and liquid level by measuring the time from transmitting to the starting point of the received wave, or to the peak of the first cycle. High precision depends on accurate ultrasonic propagation time, therefor, it is very important how judging the starting point of the received wave accurately[3]. However, precise ultrasonic propagation time is gotten hardly by circuit method when ultrasonic signal is very weak or interference signal is strong, because interference signal is filtered from received signal hardly.

It was an effective method that digital signal processing depending on the high-powered DSP chip. Noise signal was filtered by IIR bandpass digital filter in this method, the first cycle of received wave was found after significant change in fluctuation of ultrasonic signal sample was checked using the hypothesis testing, then the starting point of the received wave was determined.

\section{SCHEME DESIGN}

DSP chip was chosen as the system's processor, as its powerful processing capabilities and high running speed were beneficial to ultrasonic signal processing. The ultrasonic signal was transmitted while transducer was excited by power amplified $40 \mathrm{kHz}$ pulse from the DSP chip inner timer. The signal was received, amplified, and converted to digital signal by AD converter. The starting point of the received wave could be distinguished by the hypothesis testing after the interference noise in digital signal was filtered by IIR bandpass digital filter.

Ultrasonic is a sound wave and its propagation speed is related to air density, the larger the density, the more rapid the speed. And air density is correlated with temperature, sound velocity changes with temperature variation. All those make inaccurate measurement[4], thus temperature compensation is needed to avoid measurement error. Digital temperature sensor could be used to temperature compensation, such as DS18B20 chip of DALLAS company. Scheme design is shown in Fig.1.

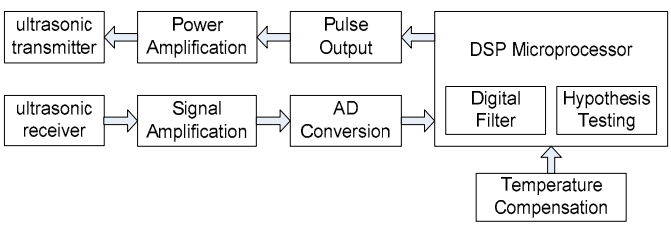

Figure 1. Scheme design

\section{CIRCUIT DESIGN}

Ultrasonic signal could be transmitted and received by ultrasonic transducer. The ultrasonic echo signal was amplified by amplifier OP27 and processed by microprocessor after completing analog to digital conversion.

Ultrasonic transmitting and receiving circuits were the core structure of the measuring circuit and those were designed as the single transducer monolithic transmit and receive circuit. Circuit design is shown in Fig. 2.

The $40 \mathrm{kHz}$ transducer was selected because ultrasonic waves could be well coupled in the air. Transducers transmitted ultrasonic signals with a high-voltage pulse excitation. The transducer has a transmitting circuit as shown in Fig.2 instead of using analog switches that lead to lower performance though simplifying the circuit.

The $40 \mathrm{kHz}$ exciting pulse was produced by the timer in DSP chip and exported to the grid electrode of the NMOS FET Q1. In the circuit, with the level of P0.22 OUT low, the NMOS FET Q1 was off, and the voltage UGS of the PMOS FET tube Q2 was nearly zero leading to Q2 off, so low level was outputted. With P0.22 OUT high, Q1 was ducted, and the grid-voltage of Q2 was divided by resistors R6 and R7. Q2 was on with UGS lower than threshold voltage, and high 
level was outputted. Hence, the positive high-voltage pulses could be generated by control of P0.22 OUT to excite the transducer.

In the receiving circuit, the current limiting resistor and two Schottky diodes confined the input voltage within about $0.4 \mathrm{~V}$ to prevent high voltage from in to the receiving circuit but had no effect on the slight received signals. The noninverting amplifier amplified the received signals about 200 times to acquire more information. Zero level was adjusted to fit for the input range of the $\mathrm{A} / \mathrm{D}$ converter. The direct current component in the ultrasonic echo signal was filtered out by capacitance $\mathrm{C} 20$.

By using the high-speed 8-bit A/D converter ADS930, each $A / D$ conversion can be accomplished within a single CLK cycle. Read of A/D converter values and control of the circuit and process of data are all performed by the DSP system that was comprised of TMS320C32.

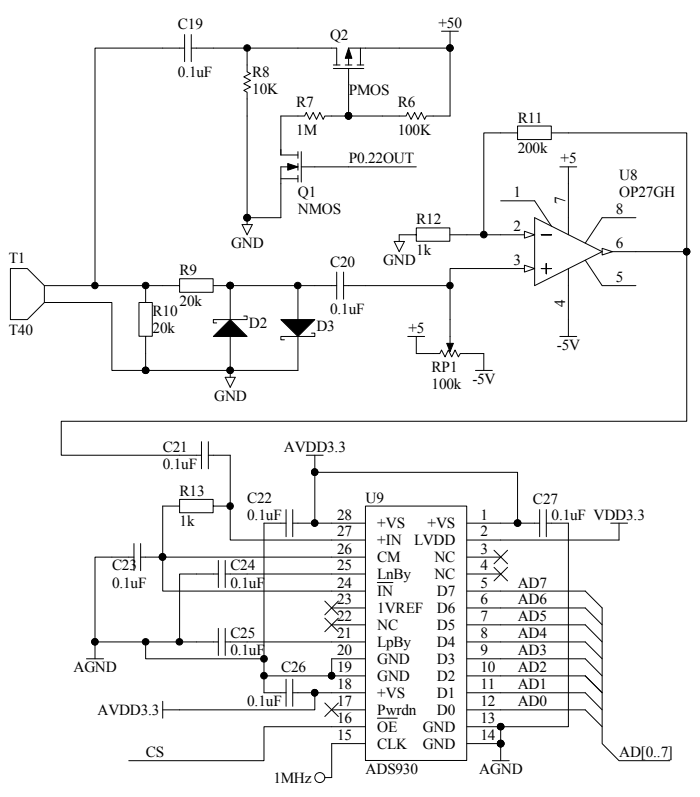

Figure 2. Circuit design

\section{Digital FILTERING}

Digital signal processing method was used to judge the ultrasonic propagation time. The processed signal was the output of a receiving transducer varying with time from the beginning of transmitting.

The received signals were amplified about 200 times to accurately locate the start point of the received wave, while the noise was also amplified and clipping distortion occured near the peak of the envelope. Fig. 3 shows that the first cycle of received wave is very weak and its amplitude is near the noise signal, it is resulted that the starting point of the received wave is determined difficultly.

The received signal was in the narrow frequency band with a slight frequency shifting for the Doppler effect, and the beginning signal frequency was a little higher than the transmitting signal. At the working site, due to the existence of background and circuit noises, power line frequency interference and offset voltage, the received signals should be filtered to decrease noise and diminish DC component.

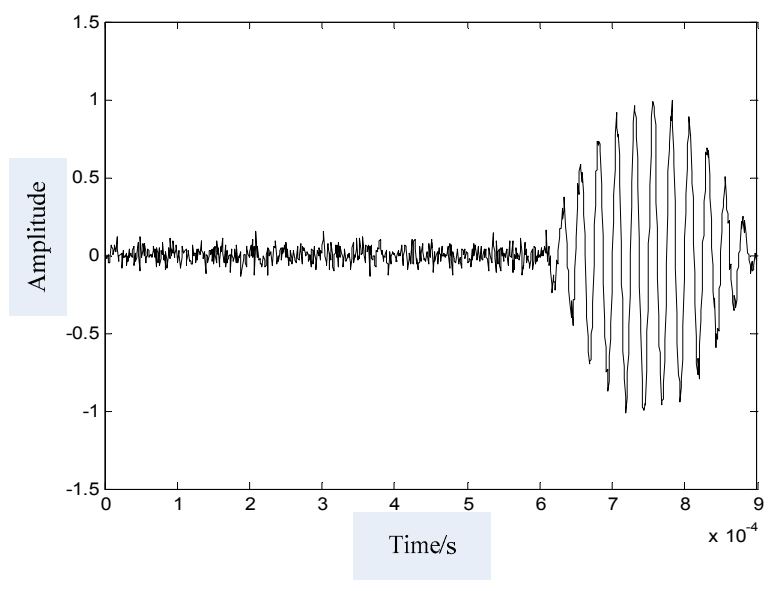

Figure 3. The received ultrasonic signal wave

It is an important step to determine a realizable transfer function $G(z)$ which approaches to specified frequency response in digital filter design, the process of determining function $G(z)$ is the digital filter design. $G(z)$ must be stable if the Infinite impulse response digital filter (IIR filter) is designed[5].

The response of the IIR filter is infinite impulse sequence. IIR filter adopts recursive structure with feedback loop. The design of IIR digital filter can draw lessons from the mature analog filter achievements, such as butterworth filter, chebyshev filter and elliptic filter.

Bilinear transformation method was a mature program in IIR digital filter design. The technical indexes of filter were determined firstly, the analog filter was designed based on the technical indexes secondly, the transfer function of analog filter became that of digital filter by bilinear transformation method at last. Analog filter design process was tedious with the large calculation amount. Matlab could be used to determine the transfer function of filter[6][7]. Matlab Link for CCS could connect Matlab, CCS development environment and DSP chip mutually, and made the development process easy.

Elliptic IIR bandpass filter is adopted in this paper because the order of elliptic filter is the smallest in the same index and the performance of elliptic filter is the best in the same order. The transfer function of filter is

$$
H(z)=\frac{B(z)}{A(z)}=\frac{b(1)+b(2) z^{-1}+\cdots+b(n+1) z^{-n}}{1+a(2) z^{-1}+\cdots+a(n+1) z^{-n}}
$$

The technical indexes of filter were set as the pass band frequency range $[35 \mathrm{kHz}, 45 \mathrm{kHz}]$, the passband ripple 0.01 $\mathrm{dB}$, stopband attenuation $60 \mathrm{~dB}$ and the sampling frequency $1 \mathrm{MHz}$. The IIR filter could be designed with 4 orders for both high pass and low pass. The coefficients of IIR filter were $\mathrm{b}=(0.0010,-0.0076,0.0248,-0.0474,0.0584,-0.0474$, $0.0248,-0.0076,0.0010), \mathrm{a}=(1.0000,-7.5792,25.3701,-$ 
48.9741, 59.6238, -46.8775, 23.2445, -6.6470, 0.8395). All the poles of the filter were within the unitcircle, and the filter was stable. Fig. 4 shows the good effect on received wave after removing the noise.

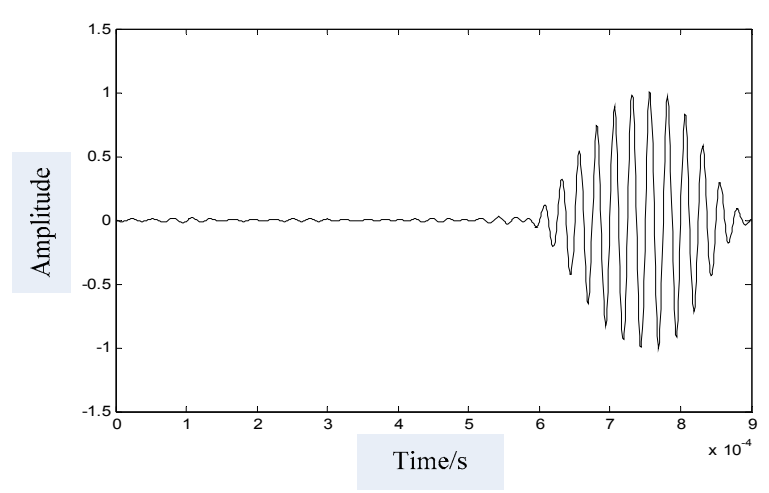

Figure 4. The filtered ultrasonic signal wave

\section{DISCRIMINATION OF THE RECEIVED WAVE START POINT}

The starting point of the received wave is the signal catastrophe point. $\chi^{2}$ testing was used to distinguish the catastrophe point after digital filtering in this paper.

$\chi^{2}$ testing could judge significant change in fluctuation of ultrasonic signal sample. The filtered ultrasonic signal is a set of time series. The point A of received wave is chosen by given obvious threshold firstly, the point $\mathrm{B}$ which is the nearest zero-crossing in front of point $\mathrm{A}$ is determined. Threshold should be positive in order that point B is the start of wave cycle. It is necessiary that the starting point of received wave lies in 2 3 cycles ahead of point $B$. The point $\mathrm{C}$ which lies in 5 cycles ahead of point $\mathrm{B}$ is chosen and the starting point is certain in $2 \sim 3$ cycles behind point $\mathrm{C}$. The two adjacent cycles are compared in turn then the first cycle of received wave is distinguished by $\chi^{2}$ testing. (Fig.5)

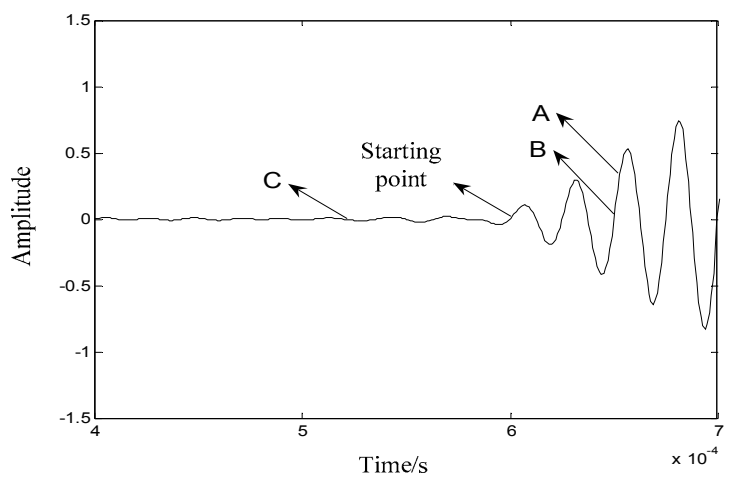

Figure 5. The schematic diagram of distinguishing the starting point
The rejection region of $\chi^{2}$ testing shows as

$$
\begin{gathered}
\frac{(n-1) S^{2}}{\sigma_{0}^{2}} \leq \chi_{1-\alpha / 2}^{2}(n-1) \\
\frac{(n-1) S^{2}}{\sigma_{0}^{2}} \geq \chi_{\alpha / 2}^{2}(n-1)
\end{gathered}
$$

Hypothesis $H_{0}: \sigma^{2}=\sigma_{0}^{2}$ and $H_{1}: \sigma^{2} \neq \sigma_{0}^{2}$ is tested at a significance level of 0.01 . There are 25 points every cycle( $n=25$ ), the results according to $\chi^{2}$ distribution table are

$$
\begin{aligned}
& \chi_{\alpha / 2}^{2}(n-1)=\chi_{0.005}^{2}(24)=45.559 \\
& \chi_{1-\alpha / 2}^{2}(n-1)=\chi_{0.995}^{2}(24)=9.886 \\
& \text { so } \\
& \frac{(n-1) S^{2}}{\sigma_{0}^{2}} \geq 45.559 \quad \frac{(n-1) S^{2}}{\sigma_{0}^{2}} \leq 9.886
\end{aligned}
$$

The two adjacent cycles are tested from point $\mathrm{C}$. The variance of the former cycle is $\sigma_{0}{ }^{2}$. It indicates that the fluctuation change of the latter cycle is significant if $H_{0}$ is refused then the latter is the first cycle. The starting point of this cycle is that of received wave. The test results of 5 cycles are shown in Tab.I.

TABLE I. THE RESUltS OF $\chi^{2}$ TESTING

\begin{tabular}{|c|c|c|c|c|c|}
\hline Cycle & $\begin{array}{c}\text { First } \\
\text { Cycle }\end{array}$ & $\begin{array}{c}\text { Second } \\
\text { Cycle }\end{array}$ & $\begin{array}{c}\text { Third } \\
\text { Cycle }\end{array}$ & $\begin{array}{c}\text { Fourth } \\
\text { Cycle }\end{array}$ & $\begin{array}{c}\text { Fifth } \\
\text { Cycle }\end{array}$ \\
\hline $\mathrm{S} 2$ & & $4.40 \mathrm{E}-04$ & $4.78 \mathrm{E}-04$ & 0.0138 & 0.0689 \\
\hline$\sigma_{0}^{2}$ & $2.70 \mathrm{E}-04$ & $2.70 \mathrm{E}-04$ & $4.23 \mathrm{E}-04$ & $4.59 \mathrm{E}-04$ & 0.0133 \\
\hline
\end{tabular}

The values of the second and third cycles are outside the rejection region while the value of the fourth cycle is inside the rejection region and much larger than critical value. Therefore, the fourth cycle is the first cycle of received wave and that starting point is the signal catastrophe point.

\section{SUMMARIES}

The starting point of ultrasonic signal was found accurately by digital filtering and hypothesis testing based on special DSP chip. The flexible numerical treatment method could be applied to measure ultrasonic propagation time precisely.

\section{ACKNOWLEDGMENT}

The research is sponsored by the National Natural Science Foundation of China (Grant No. 40976062 ).

\section{REFERENCES}

[1] LI Guang-feng; LIU Fang; GAO Yong. Travel time difference method of the ultrasonic flowmeter. Electrical Measurement \& Instrumentation [J], 2000.9, PP:13-19

[2] JIAO Bing; TANG Hui-qiang. Design of ultrasonic liquid level detecting instrument based on ARM, Instrument Technique and Sensor[J], 2008.9, PP21-23. 
[3] TANG Hui-qiang; HUANG Wei-yi; LI Ping et al. Ultrasonic wind velocity measurement based on DSP. Journal of Southeast University(English Edition) [J], 2005.1, PP:20-23

[4] GONG Zhi-yong; CHENG Yuan; GOU Yong-hua et al.. Development of an Ultrasonic Liquidometer Based on DS18B20 Temperature Sensor. measurement \& control technology [J], 2004.11, PP:6-7
[5] Sanjit K. Mitra.Digital Signal Processing A Computer-Based Approach Third Edition. Publishing House of Electronics Industry, 2006.

[6] FANG Gang; REN Xiao-hong; HE Ying-guang; WANG Tian-wen. Design and Simulation of IIR Filter Based on Matlab and DSP. Techniques of Automation and Applications [J], 2010.7, PP:42-44

[7] LIU Feng-jue; WU Jian-tong; LIU Li. IIR Digital Filter Design Based on the Bilinear Transformation and Matlab Simulation. Techniques of Automation and Applications [J], 2008.9, PP:44-47 